Research, Society and Development, v. 9, n. 11, e499119274, 2020

(CC BY 4.0) | ISSN 2525-3409 | DOI: http://dx.doi.org/10.33448/rsd-v9i11.9274

Desenvolvimento, caracterização físico-química e análise sensorial de pães integrais adicionados de resíduo da fabricação de cerveja estilo Pilsen e Porter

Development, physico-chemical characterization and sensory analysis of whole grain breads added from Pilsen and Porter style brewing residue

Desarrollo, caracterización físico-química y análisis sensorial de panes integrales agregados de residuos de elaboración de cerveza estilo Pilsen y Porter

Recebido: 14/10/2020 | Revisado: 21/10/2020 | Aceito: 30/10/2020 | Publicado: 03/11/2020

\title{
Caroline Tombini
}

ORCID: https://orcid.org/0000-0002-7543-1003

Universidade Comunitária da Região de Chapecó, Brasil

E-mail: caroline.tombini@unochapeco.edu.br

Jéssica Dall Agnol

ORCID: https://orcid.org/0000-0001-9528-6045

Universidade Comunitária da Região de Chapecó, Brasil

E-mail: jessicadallagnol@unochapeco.edu.br

Letícia Capelezzo

ORCID: https://orcid.org/0000-0003-4720-7896

Universidade Comunitária da Região de Chapecó, Brasil

E-mail: leti_capelezzo@unochapeco.edu.br

Janayne Sander Godoy

ORCID: https://orcid.org/0000-0003-2211-8643

Universidade Comunitária da Região de Chapecó, Brasil

E-mail: jgodoy@unochapeco.edu.br

Francisco Roberto da Silva Machado Junior

ORCID: https://orcid.org/0000-0002-0960-2927

Universidade Federal do Rio Grande, Brasil

E-mail: franciscojr_ea@yahoo.com.br

Cristiano Reschke Lajús

ORCID: https://orcid.org/0000-0003-3847-9793

Universidade Comunitária da Região de Chapecó, Brasil

E-mail: clajus@unochapeco.edu.br 
Josiane Maria Muneron de Mello

ORCID: https://orcid.org/0000-0002-0450-6426

Universidade Comunitária da Região de Chapecó, Brasil

E-mail: josimello@unochapeco.edu.br

Francieli Dalcanton

ORCID: https://orcid.org/0000-0003-0065-1279

Universidade Comunitária da Região de Chapecó, Brasil

E-mail: fdalcanton@unochapeco.edu.br

\section{Resumo}

A geração de resíduos pela agroindústria pode configurar um problema ambiental. O bagaço de malte, originado em grande quantidade na produção de cerveja, corresponde até a $85 \%$ dos resíduos gerados nesse processo e tem como principal destino a alimentação animal ou aterros sanitários. Devido sua constituição nutricional, pode ser utilizado na elaboração de alimentos para consumo humano. Nesse sentido, buscou-se desenvolver e avaliar pães elaborados a partir do resíduo de malte proveniente da produção de cervejas estilo Pilsen e Porter. Para isso, os pães foram produzidos utilizando o bagaço de malte, seguindo uma formulação previamente determinada e, posteriormente avaliados em relação aos parâmetros de umidade, $\mathrm{pH}$, cinzas, proteínas, lipídios, fibra bruta e carboidratos totais. Ainda, os pães foram analisados sensorialmente, onde caracterizou-se o perfil dos provadores, a preferência entre os produtos elaborados, a aceitação por escala hedônica e a intenção de compra. Como resultados principais, os pães apresentaram valores importantes nutricionalmente, com atenção para fibras, que variou de 2,15 a 2,20\% para Porter e Pilsen e proteínas, de 9,31 a $11 \%$, respectivamente. $\mathrm{Na}$ análise sensorial, não houve diferença estatística na avaliação de preferência. Os pães obtiveram notas médias acima de 6 (gostei ligeiramente) para todos os atributos avaliados e a intenção de compra demonstrou que $37 \%$ dos julgadores comprariam ambos os produtos elaborados. Dessa forma, a elaboração de pães a partir de resíduo da indústria de cerveja mostrou-se como uma opção promissora para dar uma destinação mais nobre para esse resíduo, além de apresentar-se nutricionalmente positiva. Palavras-chave: Bagaço de malte; Pães integrais; Composição centesimal; Aceitação sensorial. 


\section{Abstract}

The generation of waste by the agribusiness can be an environmental problem. Malt bagasse, originated in large quantities in beer production, corresponds to up to $85 \%$ of the residues generated in this process and has as its main destination animal feed or landfills. Due to its nutritional constitution, it can be used in the preparation of food for human consumption. In this sense, we sought to develop and evaluate breads made from malt residue from the production of Pilsen and Porter style beers. For this, the breads were produced using malt bagasse, following a previously determined formulation and subsequently evaluated in relation to the parameters of humidity, $\mathrm{pH}$, ash, proteins, lipids, crude fiber and total carbohydrates. Still, the breads were analyzed sensorially, where the profile of the tasters was characterized, the preference among the elaborated products, the acceptance by hedonic scale and the purchase intention. As main results, the breads showed nutritionally important values, with attention to fibers, which ranged from 2.15 to $2.20 \%$ for Porter and Pilsen and proteins, from 9.31 to $11 \%$, respectively. In the sensory analysis, there was no statistical difference in the preference assessment. The breads obtained average grades above 6 (I liked it slightly) for all the attributes evaluated and the purchase intention showed that $37 \%$ of the judges would buy both elaborated products. Thus, the preparation of bread from waste from the beer industry proved to be a promising option to give a more noble destination to this residue, in addition to being nutritionally positive.

Keywords: Malt bagasse; Whole grain breads; Centesimal composition; Sensory acceptance.

\section{Resumen}

La generación de residuos por parte de la agroindustria puede ser un problema ambiental. El bagazo de malta, originado en grandes cantidades en la producción de cerveza, corresponde hasta al $85 \%$ de los residuos generados en este proceso y tiene como principal destino la alimentación animal o los vertederos. Por su constitución nutricional, se puede utilizar en la preparación de alimentos para consumo humano. En este sentido, buscamos desarrollar y evaluar panes elaborados a partir de residuos de malta de la producción de cervezas estilo Pilsen y Porter. Para ello, los panes se elaboraron utilizando bagazo de malta, siguiendo una formulación previamente determinada y posteriormente evaluados en relación a los parámetros de humedad, $\mathrm{pH}$, cenizas, proteínas, lípidos, fibra cruda y carbohidratos totales. Aún así, los panes fueron analizados sensorialmente, donde se caracterizó el perfil de los catadores, la preferencia entre los productos elaborados, la aceptación por escala hedónica y la intención de compra. Como principales resultados, los panes mostraron valores 
nutricionalmente importantes, con atención a las fibras, que variaron de 2,15 a 2,20\% para Porter y Pilsen y proteínas, de 9,31 a 11\%, respectivamente. En el análisis sensorial, no hubo diferencia estadística en la evaluación de preferencias. Los panes obtuvieron calificaciones promedio superiores a 6 (me gustó un poco) para todos los atributos evaluados y la intención de compra mostró que el 37\% de los jueces compraría ambos productos elaborados. Así, la elaboración de pan a partir de residuos de la industria cervecera resultó ser una opción prometedora para darle un destino más noble a este residuo, además de ser nutricionalmente positivo.

Palabras clave: Bagazo de malta; Panes integrales; Composición centesimal; Aceptación sensorial.

\section{Introdução}

A grande geração de resíduos industriais é um problema para a sociedade. Diante disso, estudos e pesquisas para o desenvolvimento de formas de redução ou reutilização de resíduos estão em foco. As cervejarias produzem uma grande quantidade de resíduos, sendo que $85 \%$ destes correspondem ao bagaço de malte (Aliyu \& Bala, 2011). Estima-se que para cada 1000 litros de cerveja produzidos, $350 \mathrm{~kg}$ de resíduo de cervejaria úmido são obtidos (Mello, 2014).

O bagaço de malte é proveniente do processo de obtenção do mosto, pela fervura do malte moído que, após a filtração, resulta em um resíduo destinado principalmente para ração animal (Aquarone, Borzani, Schmidell, \& Lima, 2001). Sua composição química pode variar de acordo com o tipo de cevada utilizada e o seu tempo de colheita, as condições a que esta foi submetida durante o processo cervejeiro (Huige, 1994; Santos, Jiménez, Bartolomé, Gómez-Cordovés, \& Del Nozal, 2003).

Este resíduo é rico em proteínas, fibras dietéticas e cinzas, com teor médio de carboidratos e baixo em lipídios. Além disso, é considerado fonte de compostos bioativos (Almeida, 2014). Assim, o bagaço de malte pode enriquecer nutricionalmente os alimentos. $\mathrm{O}$ desenvolvimento de diferentes produtos enriquecidos com o bagaço de malte, pode gerar um novo produto com alto teor de fibras e proteínas, beneficiando a saúde do consumidor, além de gerar menos impacto para o meio ambiente e menos custos para a indústria (Mello \& Mali, 2014; Bieli et al., 2015).

Com o crescimento da consciência do consumidor sobre o uso de alimentos saudáveis, ricos em fibras, há a necessidade de desenvolver alimentos com elevado grau de fibras 
(Stojceska \& Ainsworth, 2008). A elaboração de novos produtos na área de panificação, utilizando farinhas mistas, muitas vezes advindas de resíduos da indústria, que apresentem componentes que incrementem os teores de fibras e/ou proteínas, ou possua algum componente funcional no produto final tem sido alvo de investigação de muitos pesquisadores (Morguete, Bezerra, Cordova, \& Rigo, 2011; Ktenioudaki, Chaurin, Reis, \& Gallagher, 2015; Almeida et al., 2020; Medeiros, dos Santos, Ferreira, Viana, \& Machado, 2020; Oliveira \& Andrade, 2020).

A Organização Mundial da Saúde recomenda um consumo superior a $25 \mathrm{~g} / \mathrm{dia}$ de fibra total para prevenção de doenças crônicas (OMS, 2003). Vários tipos de fibras podem ser incorporados a produtos panificados. De acordo com Stauffer (1990), fibras são adicionadas a pães por dois motivos principais, para aumentar o teor de fibra alimentar e para o decréscimo do conteúdo calórico desses pães. Um dos principais constituintes dos pães integrais são as fibras alimentares e, ao aproveitar subprodutos agroindustriais ricos em fibra, possibilita a incorporação deste nutriente, além de promover a inovação em produtos de panificação (Santos et al., 2018).

O estudo realizado por Oliveira et al. (2020) apresentou uma revisão da literatura no que diz respeito a utilização de farinhas alternativas para uso em produtos panificados. Foi evidenciado pelos autores que inúmeras farinhas podem ser incorporadas em pães sem comprometer a qualidade final dos produtos e ainda, agregar benefícios à saúde do consumidor.

Dessa forma, visando aproveitar o potencial nutritivo do bagaço de malte que é o principal subproduto do processo de produção de cerveja, este trabalho teve como objetivo elaborar dois pães adicionados de bagaço de malte de dois tipos, Porter e Pilsen, bem como a avaliação de sua composição centesimal e análise sensorial.

\section{Metodologia}

Os bagaços de malte tipo Pilsen e tipo Porter utilizados para o desenvolvimento dos pães foram doados por uma cervejaria local e armazenados congelados até a elaboração dos pães. A elaboração das formulações, bem como as análises de caracterização físico-químicas e sensoriais foram realizadas nas dependências da Universidade Comunitária da Região de Chapecó - Unochapecó, nos laboratórios de Tecnologia de Alimentos, Bromatologia, Química e Análise Sensorial.

Os demais ingredientes necessários para a formulação dos pães foram adquiridos em 
mercado local na cidade de Chapecó/SC, sendo eles: farinha de trigo integral (marca Panfácil), sal (marca Cisne), açúcar mascavo (marca Sulmel), leite integral (marca Aurora), ovos, fermento (marca Fleischmann) e óleo de coco (marca Copra).

Os bagaços de malte utilizados para a elaboração dos pães não foram submetidos ao processo de secagem, a fim de aproveitar a água presente no mesmo para a elaboração das formulações. Estes podem ser observados na Figura 1 - bagaço de malte tipo Pilsen e Figura 2 - bagaço de malte tipo Porter.

Figura 1 - Bagaço de malte tipo Pilsen.

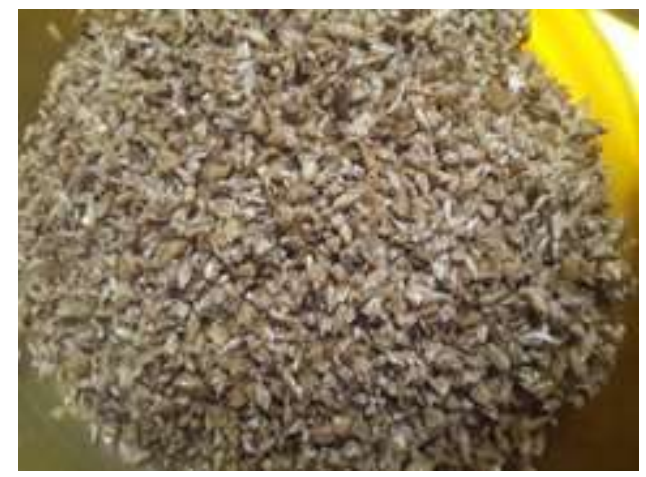

Fonte: Elaborada pelos autores.

Figura 2 - Bagaço de malte tipo Porter.

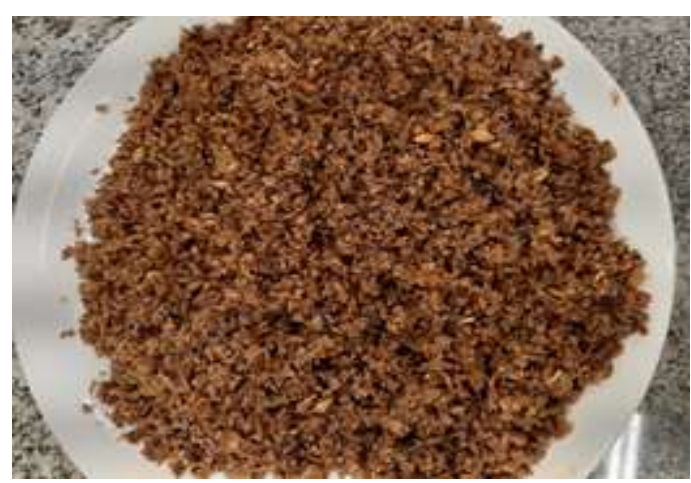

Fonte: Elaborada pelos autores.

O resíduo de bagaço de malte de ambos os tipos, Porter e Pilsen, úmidos, foram moídos em liquidificador (marca Philips Walita, modelo Liqfaz) para diminuir a sua granulometria e facilitar a homogeneização com os demais ingredientes da receita de formulação dos pães. O desenvolvimento da formulação ideal dos pães se deu através da realização de testes preliminares, até obter-se a melhor combinação e quantidade dos ingredientes. Para a elaboração dos pães, seguiu-se as formulações apresentadas na Tabela 1, 
sendo que F1 corresponde ao pão elaborado a partir do bagaço de malte da produção de cerveja estilo Pilsen e F2 ao pão obtido utilizando-se bagaço de malte oriundo da produção de cerveja Porter.

Tabela 1 - Formulação dos pães a partir de diferentes bagaços de malte.

\begin{tabular}{ccc}
\hline Ingredientes & F1 (\%) & F2 (\%) \\
\hline Bagaço de malte & 29,4 & 30,4 \\
Farinha integral & 42,2 & 48,7 \\
Leite integral & 12,7 & 10,4 \\
Ovos & 6,1 & 4,1 \\
Açúcar mascavo & 4,3 & 2,9 \\
Fermento & 2,8 & 1,8 \\
Óleo de coco & 1,8 & 1,0 \\
Sal & 0,7 & 0,7 \\
\hline
\end{tabular}

Fonte: Elaborada pelos autores.

Os pães foram elaborados com a mistura manual dos ingredientes, sendo por último adicionadas as farinhas integrais e o bagaço de malte, em que cada pão foi preparado com a adição de um tipo de bagaço de malte (Pilsen e Porter). Amassou-se as misturas até estas ficarem homogêneas e elas foram acondicionadas em formas retangulares, permanecendo em repouso para crescimento até ambas dobrarem de tamanho, por aproximadamente 1 hora.

$\mathrm{O}$ forno (marca Fischer) foi previamente aquecido a $180{ }^{\circ} \mathrm{C}$ por 5 minutos. Os pães foram assados por aproximadamente 40 minutos, até atingirem coloração característica. Após o resfriamento, estes foram acondicionados em sacos plásticos e congelados até a realização das análises físico-químicas.

A umidade, o pH e o teor de cinzas foram determinados pelos métodos descritos pelo Instituto Adolfo Lutz (Instituto Adolfo Lutz, 2008). A análise de umidade foi realizada pelo método gravimétrico, baseando-se na perda de peso do material submetido ao aquecimento em estufa (marca Nova ética $-402 / 3 \mathrm{~N}$ ), a $105{ }^{\circ} \mathrm{C}$ até peso constante. $\mathrm{O} \mathrm{pH}$ foi determinado seguindo a metodologia proposta pelo Instituto Adolfo Lutz, com o auxílio de um pHmetro Pro Linelab (marca Akso). O teor de cinzas foi determinado por incineração do material em mufla (marca Quimis), a $550{ }^{\circ} \mathrm{C}$, de acordo com a metodologia proposta.

Já o percentual de proteína bruta foi obtido pela determinação da porcentagem de nitrogênio total da amostra, segundo o método micro-Kjeldahl, conforme metodologia 
descrita pelo Ministério da Agricultura, Pecuária e Abastecimento (MAPA, 2014). Para a determinação do teor lipídeos, seguiu-se a metodologia Weende, proposta pelo equipamento determinador de gordura Goldfish TE-044-5/50 (marca Tecnal), utilizando éter de petróleo (marca Dinâmica) como solvente orgânico.

A quantificação de fibra bruta foi determinada pelo método de extração em ebulição, após uma digestão ácida e outra alcalina. A primeira extração foi com solução de $\mathrm{H}_{2} \mathrm{SO}_{4}$ (marca Dinâmica) $(1,25 \%$ p/v) por 30 minutos, seguida de filtração e lavagem. A segunda extração foi com $\mathrm{NaOH}$ (marca Dinâmica) $(1,25 \%$ p/v) por mais 30 minutos, seguida por filtração e lavagem, após secagem a $105^{\circ} \mathrm{C}$, até peso constante (Instituto Adolfo Lutz, 2008). Por último, o teor de carboidratos totais foi estimado por diferença, subtraindo de $100 \mathrm{o}$ somatório de proteínas, lipídios, cinzas e umidade, sendo o resultado expresso em percentual, seguindo o que preconiza a Resolução n. 360, de 23 de dezembro de 2003, adaptada com base na metodologia proposta pelo INFOODS - International Network of Food Data Systems (Rede Internacional de Sistemas de Dados de Alimentos), do FAO - Food and Agriculture Organization (Organização das Nações Unidas para Alimentação e Agricultura) (Maclean et al., 2003). Todas as análises físico-químicas foram realizadas em triplicata.

As análises sensoriais dos pães elaborados foram realizadas através das respostas subjetivas ou afetivas às perguntas aplicadas em um grupo de avaliadores não treinados. Realizou-se questionário de análise de perfil dos avaliadores e as análises sensoriais seguiram as metodologias do teste de preferência pareado e do teste de aceitação por escala hedônica de 9 pontos para avaliação dos atributos de aparência, aroma, sabor, textura e impressão global (em que 1 = desgostei muitíssimo; 2 = desgostei muito; 3 = desgostei moderadamente; $4=$ desgostei ligeiramente; 5 = nem gostei/nem desgostei; 6 = gostei ligeiramente; 7 = gostei moderadamente; 8 = gostei muito; 9 = gostei muitíssimo), além da avaliação de intenção de compra de 3 pontos (compraria a amostra preferida, compraria as duas amostras ou não compraria nenhuma das amostras) (Instituto Adolfo Lutz, 2008).

Este estudo foi aprovado pelo Comitê de Ética em Pesquisa em Seres Humanos da Universidade Comunitária da Região de Chapecó - Unochapecó, com o Certificado de Apresentação para Apreciação Ética (CAAE) número 20276618.9.0000.0116.

Os resultados dos testes de caracterização físico-química e da avaliação sensorial foram submetidos à análise de variância (ANOVA) no software Statistica 12.0 (StatSoft@, USA) e ao Teste de Tukey ao nível de 5\% de significância, para verificar diferenças estatísticas entre as amostras. 


\section{Resultados e Discussão}

As formulações F1 e F2 elaboradas para os pães contam, respectivamente, com $29,37 \%$ e $30,45 \%$ de bagaço de malte e os produtos finais obtidos podem ser observados nas Figuras 3 e 4, respectivamente. O percentual de bagaço utilizado na elaboração dos pães é interessante, pois percebe-se que houve um alto percentual de substituição da farinha integral tradicional. Quantidade semelhante de incorporação em pães foi utilizado pelo estudo realizado por Mattos (2010), que estudou a fabricação de pão de forma utilizando $30 \%$ de bagaço de malte úmido em sua formulação. Ainda, Ktenioudaki et al. (2012) avaliaram o potencial de bagaço de malte como ingrediente funcional em massa assada na forma de palito. Eles observaram que a adição de $25 \%$ e $35 \%$ bagaço de malte aumentou significativamente (p $<0,005)$ o teor de proteína do produto, sendo que a adição de $15 \%$ de bagaço de malte mais do que duplicou o teor de fibras nas amostras.

Figura 3 - Pão utilizando bagaço de malte Pilsen.

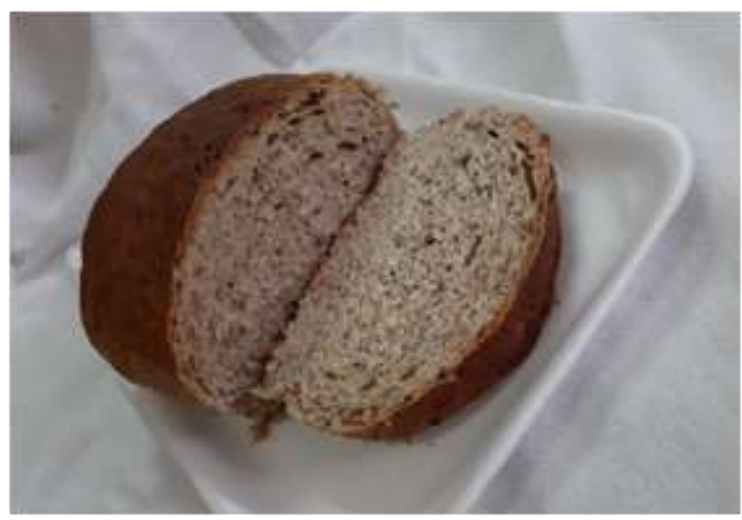

Fonte: Elaborada pelos autores.

Figura 4 - Pão utilizando bagaço de malte Porter.

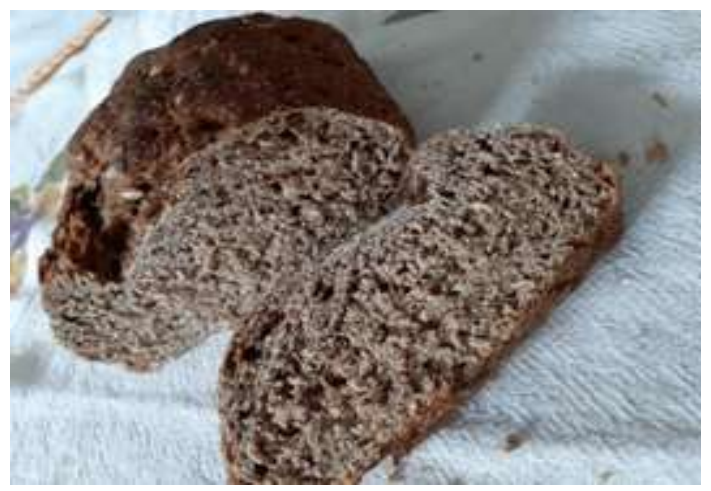

Fonte: Elaborada pelos autores. 
Com as análises físico-químicas dos pães elaborados, obtiveram-se os resultados apresentados na Tabela 2.

Tabela 1 - Caracterizações físico-químicas dos pães F1 e F2, com bagaço de malte Pilsen e Porter.

\begin{tabular}{ccc}
\hline Caracterizações & F1 & F2 \\
\hline Umidade & $34,10 \pm 3,49 \%^{\mathrm{a}}$ & $33,96 \pm 1,54 \%^{\mathrm{a}}$ \\
Cinzas & $2,47 \pm 0,13 \%^{\mathrm{b}}$ & $3,21 \pm 0,11 \%^{\mathrm{a}}$ \\
pH & $6,49 \pm 0,01^{\mathrm{b}}$ & $6,57 \pm 0,01^{\mathrm{a}}$ \\
Proteína & $11,0 \pm 0,38 \%^{\mathrm{a}}$ & $9,31 \pm 0,43 \%^{\mathrm{b}}$ \\
Lipídios & $2,42 \pm 0,27 \%^{\mathrm{a}}$ & $2,54 \pm 0,21 \%^{\mathrm{a}}$ \\
Fibra bruta & $2,20 \pm 0,01 \%^{\mathrm{a}}$ & $2,15 \pm 0,05 \%^{\mathrm{a}}$ \\
Carboidratos totais & $50,01 \% \%^{\mathrm{a}}$ & $50,98 \% \mathrm{a}$
\end{tabular}

Nota: Médias seguidas de mesma letra minúscula na linha não diferem entre si ao nível de 5\% de significância.

Fonte: Elaborada pelos autores.

O valor de umidade dos pães encontra-se próximo ao apresentado no estudo de Santos et al. (2018), que avaliou pães elaborados com farinha da casca de mamão, e obteve valores

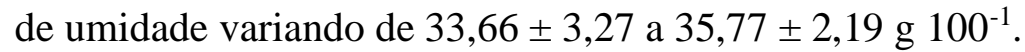

Em relação ao teor de cinzas apresentado pelos pães, estes foram superiores aos valores encontrados por Borges, Vidigal, Silva, Pirozi e Paula (2013), que avaliou pães de forma elaborados com farinha mista de trigo e quinoa, e obteve valores variando de 1,57 a

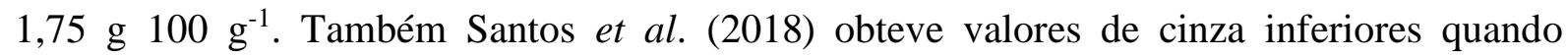

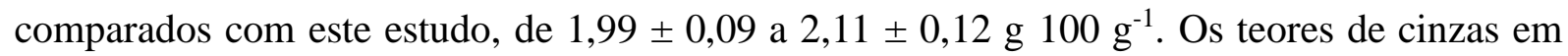
alimentos são considerados como parâmetro de qualidade, pois valores elevados indicam maiores teores de cálcio, magnésio, ferro, fósforo, sódio e outros componentes minerais (Wang \& Zheng, 2003).

$\mathrm{O}$ pH é um parâmetro importante para determinação da qualidade microbiológica do produto (Santos et al., 2018). Os valores para as amostras de pães encontram-se próximos da neutralidade, sendo este um ponto positivo, pois valores mais baixos podem ser indicativos de contaminação microbiana (Cardoso et al., 2019).

Pode-se destacar os resultados obtidos nas caracterizações de proteína e fibra bruta, onde o pão adicionado de bagaço de malte tipo Pilsen apresentou teor de proteína de 11,0 \pm 
0,38\% e para o pão adicionado de bagaço de malte tipo Porter, 9,31 $\pm 0,43 \%$. Já para fibra bruta, o pão com bagaço de malte Pilsen teve um resultado de $2,20 \pm 0,01 \%$ e para o pão com bagaço de malte Porter $2,1 \pm 0,05 \%$.

Avaliando os teores de lipídios encontrados para as amostras, observou-se que estão de acordo com os valores estabelecidos pela legislação para alimentos sólidos com baixo teor de gorduras totais, com valores inferiores a 3 g por porção (50 g) (Resolução n. 54, de 12 de novembro de 2012) e os teores de carboidratos encontram-se similares aos valores que Borges

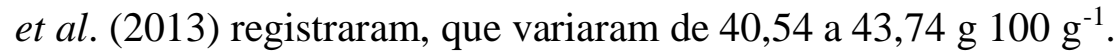

De acordo com a Tabela Brasileira de Composição de Alimentos - TACO (UNICAMP, 2011), o pão integral clássico tem 34,70 g $100 \mathrm{~g}^{-1}$ de umidade, 9,40 g $100 \mathrm{~g}^{-1}$ de proteína, 3,70 g $100 \mathrm{~g}^{-1}$ de lipídios, 49,90 g $100 \mathrm{~g}^{-1}$ de carboidratos, 2,30 g $100 \mathrm{~g}^{-1}$ de cinzas e

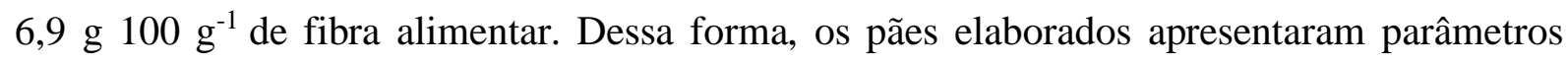
físico-químicos muito semelhantes aos apresentados pela Tabela TACO, com valores de proteína superiores para o pão elaborado a partir do bagaço de malte Pilsen, evidenciando a possibilidade de substituição da farinha integral tradicional por um resíduo da agroindústria, o bagaço de malte.

Para a análise sensorial, o público participante foi composto por estudantes, professores e funcionários da Unochapecó, não treinados. A distribuição de provadores por gênero e idade pode ser visualizado na Figura 5 e na Figura 6, respectivamente.

Figura 5 - Distribuição de participantes da análise sensorial por gênero.

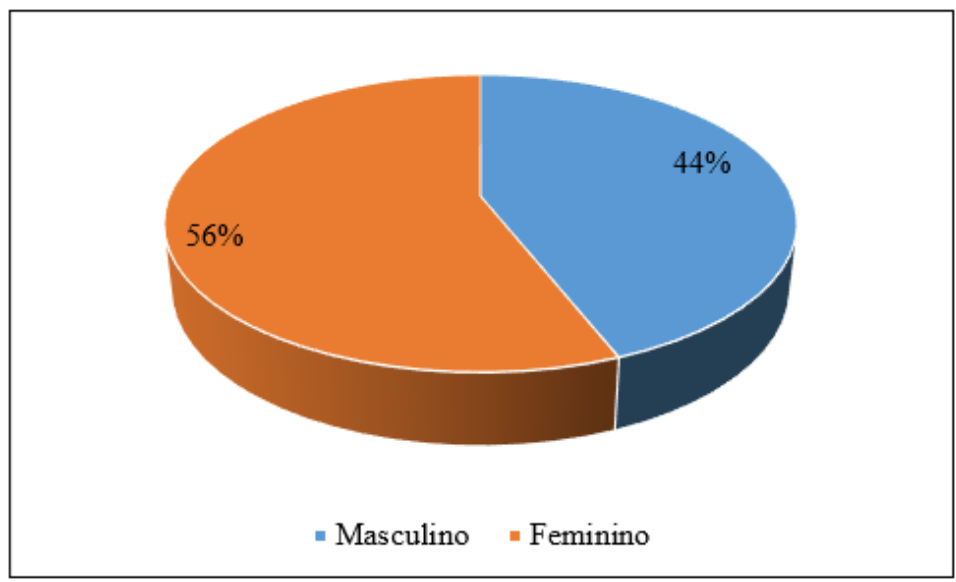

Fonte: Elaborada pelos autores. 
Figura 6 - Distribuição de participantes da análise sensorial por faixa etária.

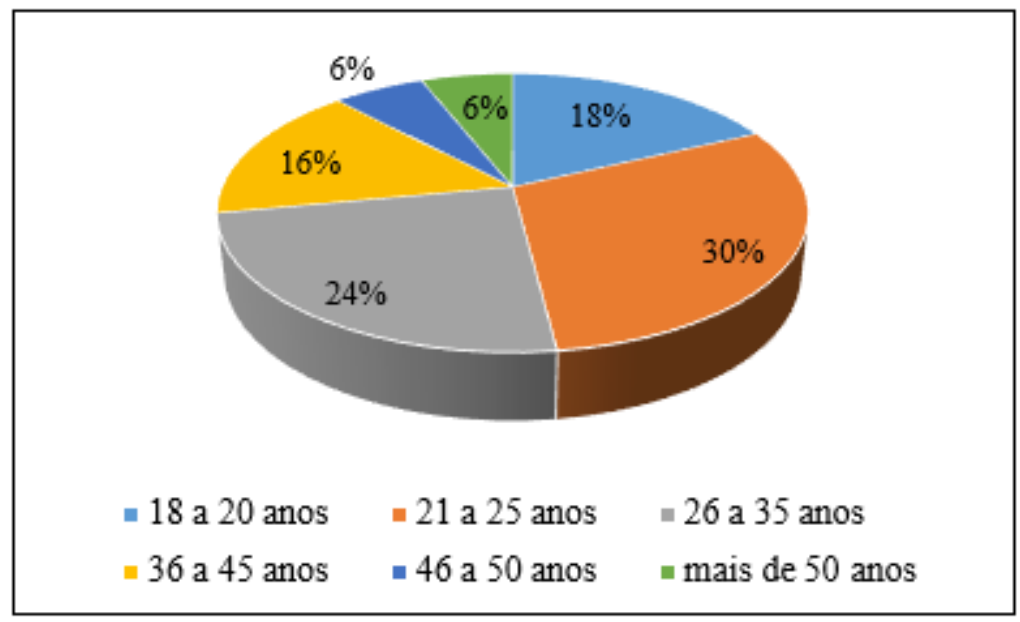

Fonte: Elaborada pelos autores.

Pode-se observar, através da Figura 5, que houve homogeneidade entre o sexo dos julgadores. A Figura 6 demonstrou-se a relação da faixa etária dos participantes, sendo que o maior percentual de julgadores pertence a faixa etária entre 21 a 25 anos representando 15 pessoas (30\%), seguido da faixa de 26 a 35 anos, que contou com 12 julgadores (24\%). O menor percentual de avaliadores pertenceu a faixa de idade de com mais de 46 anos, sendo $6 \%$ pertencente a faixa de 46 a 50 anos e outros $6 \%$ com mais de 50 anos.

Em relação a escolaridade dos avaliadores, observou-se que a maioria apresenta superior completo, seguida de superior incompleto, como pode ser observado na Figura 7.

Figura 7 - Grau de escolaridade dos provadores.

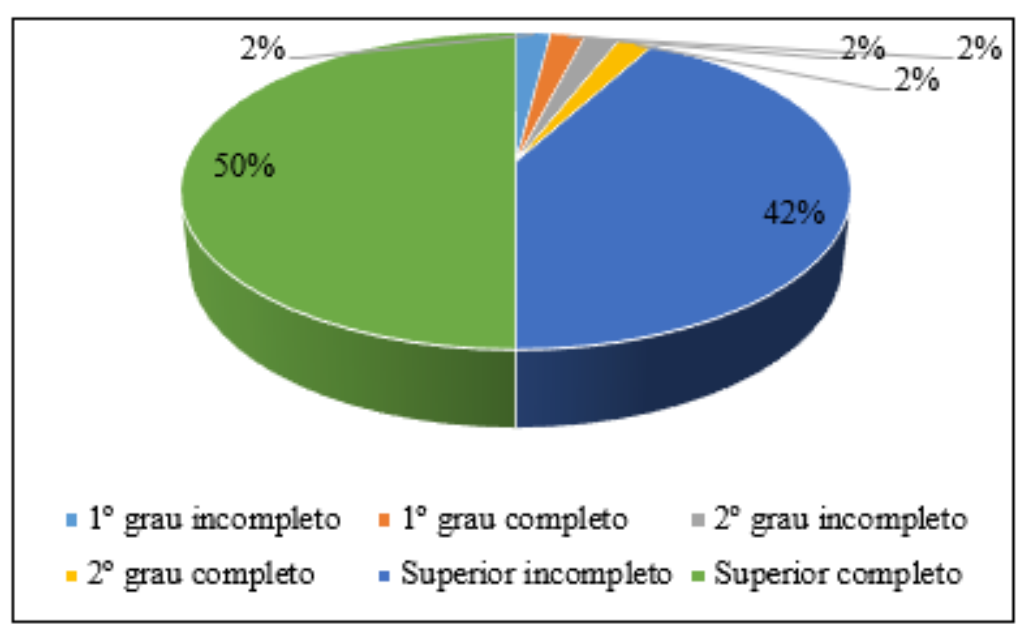

Fonte: Elaborada pelos autores. 
Como forma de caracterizar demograficamente os provadores, perguntou-se a cidade e predominantemente, observou-se como sendo Chapecó a cidade com o maior número de julgadores (Figura 8). Fato este esperado, visto que nesta mesma cidade foi onde realizou-se a coleta de dados.

Figura 8 - Cidade de residência dos julgadores.

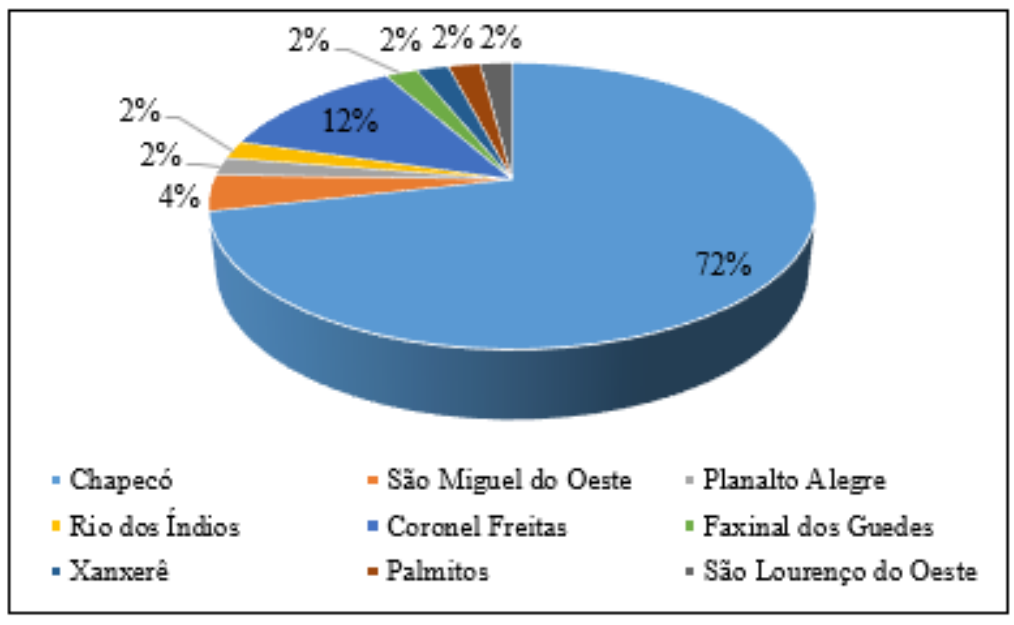

Fonte: Elaborada pelos autores.

Como forma de avaliar o perfil de consumo dos julgadores que participaram da análise sensorial, perguntou-se com que frequência os participantes consomem pão. $\mathrm{O}$ resultado obtido pode ser observado na Figura 9.

Figura 9 - Distribuição dos participantes da análise sensorial em relação à frequência de consumo de pão.

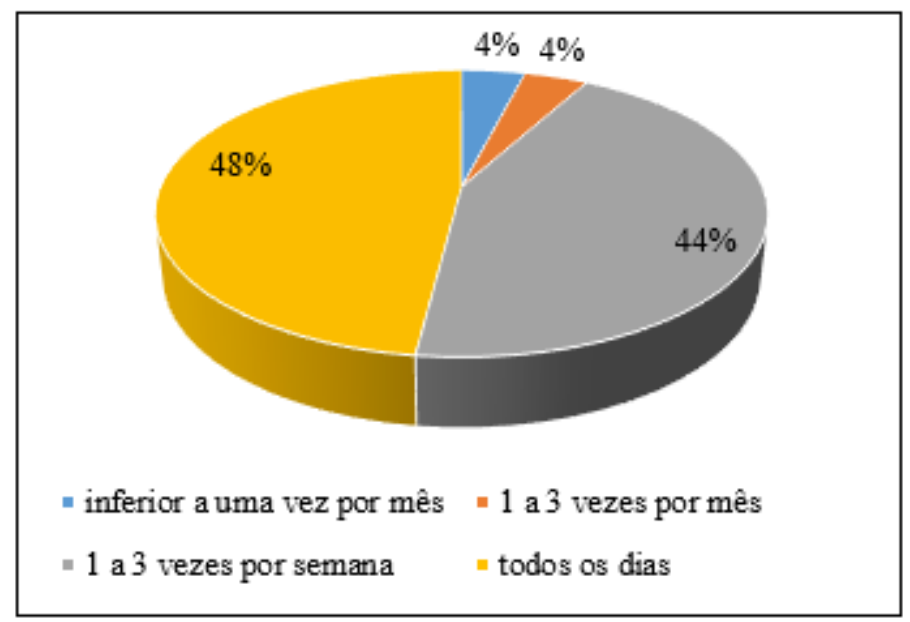

Fonte: Elaborada pelos autores. 
Observou-se que os participantes são frequentes consumidores de pão. O maior percentual de julgadores que participaram da pesquisa, correspondendo a $48 \%$, afirmaram que consomem pão todos os dias. Ainda, outros 44\% disseram que comem pão de 1 a 3 vezes por semana. Juntos, este grupo representa 92\%. Este é um valor importante para avaliar as respostas obtidas com a avaliação sensorial, pois indica que os julgadores são consumidores de pães.

Em relação ao consumo de pão integral, observou-se, de acordo com a Figura 10, que nem todos os avaliadores que consomem pão todos os dias, optam pela versão integral do produto, pois somente $18 \%$ consomem pão integral todos os dias. Cabe ressaltar que $27 \%$ dos julgadores consomem pão integral 1 a 3 vezes por semana, mas ainda assim, há àqueles que consomem numa frequência de 1 a 3 vezes por mês, correspondendo a $26 \%$ e os que menos consomem pão, correspondem a $29 \%$ dos provadores. Pode-se inferir que os consumidores que fazem a ingestão de pão, preferem ainda o pão tradicional.

Figura 10 - Distribuição dos participantes da análise sensorial em relação à frequência de consumo de pão integral.

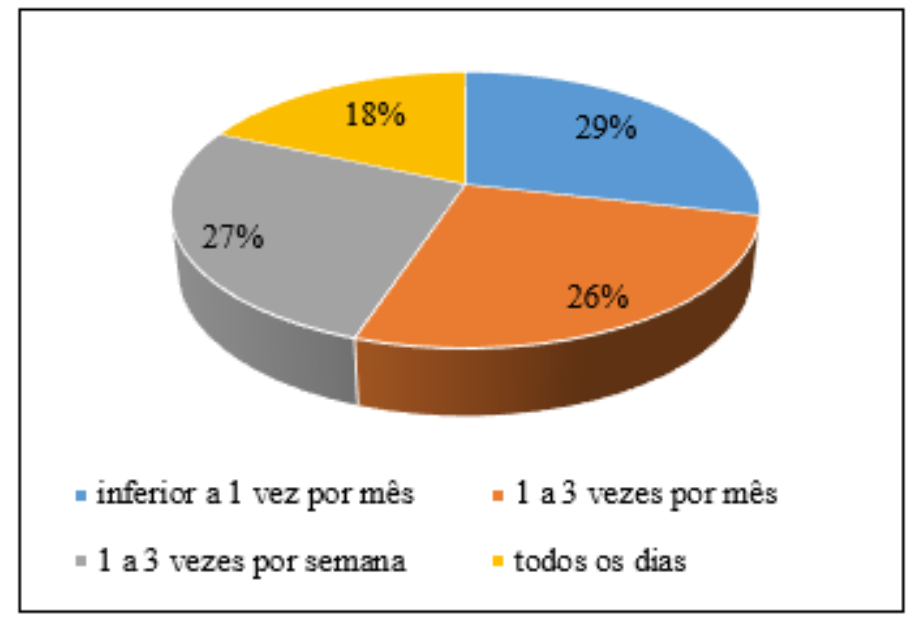

Fonte: Elaborada pelos autores.

Em um estudo realizado por Siquieri, Filbido e Bacarji (2018), buscou-se identificar o perfil de consumidores de produtos integrais em Cuiabá/MT e como resultados, observou-se que pães integrais são os produtos mais consumidos, dentro da classe de produtos integrais, pelos consumidores avaliados no estudo.

Com a avaliação sensorial de preferência realizada, obtiveram-se os resultados apresentados na Tabela 3. Cabe ressaltar que em virtude de preenchimento incorreto da ficha de avaliação, somente 45 respostas puderam ser contabilizadas para avaliação. 
Tabela 2 - Resultados do teste de análise sensorial pareado de preferência para o pão com bagaço de malte Pilsen (F1) e bagaço de malte Porter (F2).

\begin{tabular}{cc}
\hline Pão com bagaço de malte & Preferência \\
\hline F1 & 25 \\
F2 & 20 \\
\hline
\end{tabular}

Fonte: Elaborada pelos autores.

Verificou-se, através da Tabela 3, que 25 julgadores preferiram a amostra de pão com bagaço Pilsen e 20 julgadores preferiram a amostra de pão com bagaço de malte Porter. Para uma amostragem de 45 provadores no teste de preferência pareada, usando $5 \%$ de significância, a amostra preferida deveria ser indicada por no mínimo 30 provadores (Meilgaard, Civille, \& Carr, 1991). Logo, neste estudo não houve diferença significativa na preferência entre os dois pães.

A avaliação sensorial de aceitação por escala hedônica de 9 pontos para os atributos aparência, aroma, sabor, textura e impressão global, as médias e valores de desvio padrão para cada atributo, bem como a análise de Tukey encontram-se na Tabela 4.

Tabela 3 - Médias das notas atribuídas pelos julgadores para avaliação de aceitação por escala hedônica.

\begin{tabular}{ccc}
\hline Atributos & F1 & F2 \\
\hline Aparência & $7,60 \pm 1,10^{\mathrm{a}}$ & $7,04 \pm 1,61^{\mathrm{a}}$ \\
Aroma & $7,64 \pm 1,25^{\mathrm{a}}$ & $7,02 \pm 1,97^{\mathrm{a}}$ \\
Sabor & $7,11 \pm 1,25^{\mathrm{a}}$ & $6,82 \pm 1,81^{\mathrm{a}}$ \\
Textura & $6,96 \pm 1,54^{\mathrm{a}}$ & $7,11 \pm 1,73^{\mathrm{a}}$ \\
Impressão global & $7,40 \pm 1,03^{\mathrm{a}}$ & $7,04 \pm 1,66^{\mathrm{a}}$ \\
\hline
\end{tabular}

Nota: Médias seguidas de mesma letra minúscula na linha não diferem entre si ao nível de 5\% de significância

Fonte: Elaborada pelos autores.

Através da análise estatística, concluiu-se que os valores não apresentaram diferença significativa estatisticamente, assim sendo, ambos os pães elaborados se mostraram bem aceitos. Observa-se, portanto, que as notas atribuídas a ambas as amostras apresentaram-se acima de 6,8 para todos os atributos avaliados. A nota 6 significa gostei ligeiramente, e a 7, gostei moderadamente, ou seja, os produtos foram bem aceitos pelos avaliadores, 
(CC BY 4.0) | ISSN 2525-3409 | DOI: http://dx.doi.org/10.33448/rsd-v9i11.9274

evidenciando a possibilidade de substituição da farinha integral tradicional pelo resíduo proveniente da produção de cerveja.

O estudo de Mattos (2010) também realizou avaliação sensorial do pão de forma produzido com o bagaço e foi possível observar que este apresentou índice de aceitação maior do que $80 \%$ e os quesitos de impressão global, aroma, sabor, textura e cor indicam boa aceitação do pão pelos julgadores, os quais, também, indicaram que o pão apresentou aspecto de pão integral com sabor acentuado característico de levedo de cerveja.

Em relação a utilização de farinhas a partir de resíduos, o estudo de Lima et al., (2019) avaliou a adição de farinha de resíduo de graviola para elaboração de pão de forma. Sensorialmente, o estudo indicou que pães com adição de 10\% tiveram aceitabilidade de $73 \%$ e com a adição de $20 \%$ de farinha de resíduo de graviola, a aceitabilidade ficou na casa dos $66 \%$. Dessa forma, os autores concluem que substituir parcialmente a farinha de trigo pela do resíduo de graviola é uma opção com potencial mercadológico.

Carvalho et al. (2020) avaliaram a adição de farinha de casca de mamão em pães. A análise sensorial realizada demonstrou maior aceitabilidades para formulação sem o resíduo, demonstrando que embora os resíduos possam enriquecer nutricionalmente as formulações, é necessário encontrar um equilíbrio para que os atributos sensoriais também sejam interessantes ao consumidor.

Foi avaliada, por fim, a intenção de compra, em que os provadores deveriam assinalar a opção que julgavam adequada em relação a compra das amostras avaliadas, ou seja, se comprariam a amostra preferida, as duas ou nenhuma. Os resultados obtidos encontram-se na Figura 11. Nesta avaliação, considerou-se a opinião de 38 provadores, visto que houveram erros de preenchimento nas fichas de avaliação que as invalidaram para a análise. 
Figura 11 - Intenção de compra.

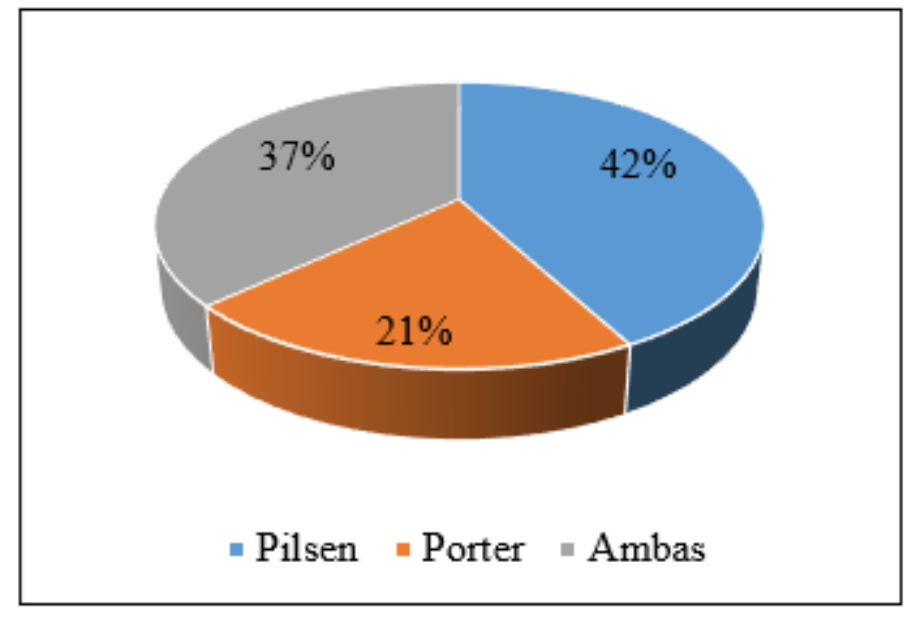

Fonte: Elaborada pelos autores.

Dessa forma, inferiu-se que $42 \%$ dos provadores comprariam o pão com bagaço de malte Pilsen, 21\% comprariam o pão com bagaço de malte Porter e $37 \%$ dos avaliadores, comprariam os dois pães elaborados. Nenhum avaliador assinalou a opção de que não compraria nenhuma das amostras.

Os pães elaborados foram bem avaliados pelos avaliadores e apresentaram características importantes nutricionalmente, demonstrando potencial de utilização para elaboração de produtos panificados. Sugere-se a avaliação da adição de percentuais superiores de bagaço de malte para verificar o limite máximo de adição deste tipo de resíduo em produtos panificados, bem como a avaliação da combinação de diferentes bagaços de malte provenientes dos mais diversos estilos de cerveja.

Cabe ressaltar ainda, que a principal limitação da utilização deste resíduo diz respeito ao seu alto teor de umidade que pode acarretar em contaminação microbiana impossibilitando seu uso. Assim, é necessário atenção a essa questão quando deseja-se estudar novas formas de utilização do bagaço de malte.

\section{Considerações Finais}

Os resultados alcançados com a caracterização físico-química demonstraram que utilizar o bagaço de malte na produção de pães é uma alternativa interessante, pois o mesmo apresentou valores satisfatórios para os teores de fibras e proteínas, que são importantes constituintes nutricionais. Ainda, comprovou-se com a análise sensorial de aceitação, que obteve notas acima de 6,8 para todos os atributos avaliados, que os pães elaborados foram 
bem aceitos pelos julgadores, e que seriam uma opção de compra. As análises realizadas não apresentaram diferença estatística significativa, exceto os valores de $\mathrm{pH}$, cinzas e proteínas.

Esses resultados se mostraram promissores, visto que até o momento as principais formas de utilização desse resíduo são para a alimentação animal ou ainda, descarte em aterros sanitários. Desta forma, pode-se concluir que o bagaço de malte pode ser utilizado para a produção de pães e ainda, pode diferenciar um produto alimentício já existente, contribuindo para o desenvolvimento de novos produtos.

\section{Agradecimentos}

À Universidade Comunitária da Região de Chapecó - Unochapecó, com bolsa de iniciação científica e bolsas de mestrado, ao Programa Institucional de Bolsas de Iniciação Científica (PIBIC/CNPq), ao Programa de Bolsas Universitárias do Estado de Santa Catarina, ao Fundo de Apoio à Manutenção e ao Desenvolvimento da Educação Superior, bem como à Dalla Cervejaria, localizada na cidade de Cordilheira Alta/SC, pela doação do resíduo de bagaço de malte para o desenvolvimento deste estudo.

\section{Referências}

Aliyu, S., \& Bala, M. (2011). Brewer's spent grain: A review of its potentials and applications. African Journal of Biotechnology, 103(3), 324-331.

Almeida, A. R. (2014). Compostos bioativos do bagaço de malte: fenólicos, capacidade antioxidante in vitro e atividade antibacteriana. Dissertação de Mestrado, Universidade Federal do Paraná, Curitiba, Brasil. Recuperado de https://acervodigital.ufpr.br/bitstre am/handle/1884/35738/R\%20-\%20D\%20-\%20ALINE\%20DA\%20ROSA\%20AL

MEIDA.pdf?sequence $=1 \&$ isAllowed=y.

Almeida, R. L., Santos, N. C., Pereira, T. dos S., Silva, V. M. de Alcântara, Ribeiro, V. H. de A., Silva, L. N., Almeida, R. D., Santos, S. B. F., Moreira, F. I. N., \& Lima, S. E. R. (2020). Composição físico-químicas de cookies adicionados de resíduos do arroz vermelho. Research, Society and Development, 9(1), e151911852, 1-16. 
Aquarone, E., Borzani, W., Schmidell, W., \& Lima, U. A. L. (2001). Biotecnologia Industrial. São Paulo: Edgar Blücher Ltda.

Bieli, B. C., Marques, D. R., Marchi, L. B., Quelhas, J. O. F., Chinellato, M. M., Monteiro, C. C. F., \& Monteiro, A. R. G. (2015). Produção de snack extrusado com adição de farinha de bagaço de malte. Revista Tecnológica, 321-326.

Borges, J. T. S., Vidigal, J. G., Silva, N. A. S., Pirozi, M. R., \& Paula, C. D. (2013). Caracterização físico-química e sensorial de pão de forma contendo farinha mista de trigo e quinoa. Revista Brasileira de Produtos Agroindustriais, 15(3), 305-319.

Cardoso, R. V. C., Fernandes, Â., Heleno, S. A., Rodrigues, P., Gonzáles-Paramás, A. M., Barros, L.; \& Ferreira, I. C. F. R. (2019). Physicochemical characterization and microbiology of wheat and rye flours. Food Chemistry, 280, 123-129.

Carvalho, J. B., Marques, K. H. D. S. R., Mesquita, A. A., Paula, G. H., Lima, M. S., Ferreira, S. V., Medeiros, J. S., Teixeira, P. C., Nicolau, E. S., \& da Silva, M. A. P. (2020). Propriedades químicas e funcionais da casca de mamão verde submetida à secagem em diferentes temperaturas e aplicação em pães. Research, Society and Development, 9(5), e29953154-e29953154, 1-25.

El-Dash, A., Cabral, L. C., \& Germani, R. (1994). Tecnologia de farinhas mistas. Brasília: Embrapa, (6).

Hoseney, R. C. (1991). Principios de Ciencia y Tecnología de los Cereales. Zaragosa, Espanha: Acribia.

Huige, N. J. (1994). Brewery by-products and effluents. In: HARDWICK, W.A. Handbook of Brewing. New York: Marcel Dekker.

Instituto Adolfo Lutz. (2008). Normas Analíticas do Instituto Adolfo Lutz: Métodos químicos e físicos para análise de alimentos. São Paulo: IMESP. 
Ktenioudaki, A., Chaurin, V., Reis, S., \& Gallagher, E. (2012). Brewer's spent grain as a functional ingredient for breadsticks. International Journal of Food Science and Technology, 47(8), 1765-1771.

Ktenioudaki, A., Alvarez-Jubete, L., Smyth, T.S., Kilcawley, K., Rai, D. K., \& Gallagher, E. (2015). Application of bioprocessing techniques (sourdough fermentation and technological aids) for brewer's spent grain breads. Food Research International, 73, 107-116.

Lima, D. V., Azevedo, O. O. C., Silva, N. S., Silva, G. S., Pontes, E. D. S., Araujo, M. G. G., Pereira, D. E., Piovesan, N., Medeiros, R. G., Soares, J. K. B., \& Viera, V. B. (2020). Desenvolvimento e avaliação sensorial de pão de forma adicionado da farinha do resíduo da graviola. Research, Society and Development, 9(2), e172911857, 1-18.

Maclean, W., Harnly, J., Chen, J., Chevassus-Agnes, S., Gilani, G., Livesey, G., \& Warwick, P. (2003). Food energy - Methods of analysis and conversion factors. Roma: FAO.

MAPA - Ministério da Agricultura, Pecuária e Abastecimento. (2014). Determinação de proteína bruta em produtos de origem animal por acidimetria, MET POA/SLAV/39/02/01. Recuperado de: http://www.agricultura.gov.br/assuntos/laboratorios/legislacoes-e-metodos/ arquivos-metodos-da-area-poa-iqa/met-poa-slav-39-02-proteina-bruta.pdf/@ @download/file/ MET\%20POA\%20SLAV\%2039\%2002\%20\%20Prote\%C3\%ADna\%20bruta.pdf.

Mattos, C. (2010). Desenvolvimento de um pão fonte de fibras a partir do bagaço de malte. Monografia de graduação, Universidade Federal do Rio Grande do Sul, Porto Alegre, RS, Brasil.

Medeiros, J. S., dos Santos, L. S., Ferreira, S. V., Viana, L. F., \& Machado, A. R. (2020). Desenvolvimento de biscoitos a partir do resíduo da extração de suco de caju do cerrado Goiano. Research, Society and Development, 9(7), e39973082, 1-14.

Meilgaard, M., Civille, G. V., \& Carr, B. T. (1991). Sensory evaluation techniques. (2a ed.), Flórida: CRC Press, 1991. 
Mello, L. R., \& Mali, S. (2014). Use of malt bagasse to produce biodegradable baked foams made from cassava starch. Industrial Crops and Products, 55, 187-193.

Mello, V. S. A. (2014). Determinação da composição do malte de cevada e estudo das suas potenciais aplicações. Monografia de graduação, Escola de Engenharia de Lorena, Lorena, SP, Brasil.

Morguete, E. M., Bezerra, J. R. M. V., Cordova, K. R. V., \& Rigo, M. (2011). Elaboração de pães com adição de farelo de soja. Ambiência, 7(3), 481-488.

Oliveira, D. R. de, \& Andrade, A. P. C. de. (2020). Elaboração de pão de forma adicionado de yacon. Research, Society and Development, 9(8), e230985481, 1-16.

Oliveira, I. M., Melo, F. D. S. N., Sousa, M. M., Sousa Menezes, M., Oliveira Paz, E., \& Silva Cavalcanti, M. (2020). Utilização de farinhas alternativas em produtos de panificação: uma revisão literária. Research, Society and Development, 9(9), e441996228, 1-27.

OMS - Organização Mundial da Saúde. (2003). World Health Organization. Diet, nutrition and the prevention of chronic diseases. Report of a WHO consultation. Geneva: World Health Organization.

Resolução n. 54, de 12 de novembro de 2012. Dispõe sobre o Regulamento Técnico sobre Informação Nutricional Complementar. Brasília, DF. Recuperado de http://portal.anvisa.g ov.br/legislacao\#/visualizar/28921.

Resolução n. 360, de 23 de dezembro de 2003. dispõe sobre a aprovação do Regulamento Técnico sobre Rotulagem Nutricional de Alimentos Embalados, tornando obrigatória a rotulagem nutricional dos mesmos, salvo exceções descritas na resolução. Brasília, DF. Recuperado de Http://portal.anvisa.gov.br/legislacao\#/visualizar/27327.

Santos, M., Jiménez, J. J., Bartolomé, B., Gómez-Cordovés, C., \& Del Nozal, M. J. (2003). Variability of brewer's spent grain within a brewery. Food Chemistry, 80(1), 17-21. 
Santos, C. M., Rocha, D. A., Madeira, R. A. V., Queiroz, E. R., Mendonça, M. M., Pereira, J., \& Abreu, C. M. P. (2018). Preparação, caracterização e análise sensorial de pão integral enriquecido com farinha de subprodutos do mamão. Brazilian Journal of Food Technology, 21 (e2017120), 1-9.

Siquieri, J. P. A., Filbido, G. S., \& Bacarji, A. G. (2018). Perfil do consumidor de alimentos integrais na cidade de Cuiabá/MT. Revista Princípia, 41, 180-189.

Stauffer, C. E. (1990). Functional additives for bakery foods. Nova Iorque: AVI Books.

Stojceska, V., \& Ainsworth, P. (2008). The effect of different enzymes on the quality of highfibre enriched brewer's spent grain breads. Food Chemistry, 110(4), 865-872.

UNICAMP - Universidade Estadual de Campinas. (2011). Tabela Brasileira de Composição de Alimentos - TACO. Versão 4. Campinas: UNICAMP. Recuperado de: http://www.cfn.org.br/wp-content/uploads/2017/03/taco_4_edicao_ampliada_e_revisada.pdf.

Wang, S. Y., \& Zheng, W. (2003). Oxygen radical absorbing capacity of phenolics in blueberries, cranberries, chokeberries, and lingonberries. Journal of Agricultural and Food Chemistry, 51(2), 873-878.

Porcentagem de contribuição de cada autor no manuscrito

$$
\begin{gathered}
\text { Caroline Tombini - } 20 \% \\
\text { Jéssica Dall Agnol }-20 \% \\
\text { Letícia Capelezzo }-10 \% \\
\text { Janayne Sander Godoy }-10 \%
\end{gathered}
$$

Francisco Roberto da Silva Machado Junior - 10\%

Cristiano Reschke Lajús - 10\%

Josiane Maria Muneron de Mello - 10\%

Francieli Dalcanton - 10\% 